\title{
Adeno-Associated Virus-Mediated Gene Transfer of Tissue Inhibitor of Metalloproteinases-1 Impairs Neutrophil Extracellular Trap Formation and Ameliorates Hepatic Ischemia and Reperfusion Injury
}

Sergio Duarte, Patrick Matian, Stacy Ma, Ronald W. Busuttil, and Ana J. Coito

From The Dumont-UCLA Transplant Center, Division of Liver and Pancreas Transplantation, Department of Surgery, David Geffen School of Medicine, University of California, Los Angeles, Los Angeles, California

Accepted for publication

May 3, 2018.

Address correspondence to Ana J. Coito, M.Sc., Ph.D., The Dumont-UCLA Transplant Center, 77-120 CHS, Box 957054, Los Angeles, CA 90095 7054. E-mail: acoito@mednet. ucla.edu.

\begin{abstract}
Matrix metalloproteinase-9 (MMP-9) is abundantly expressed by infiltrating leukocytes and contributes to the pathogenesis of hepatic ischemia and reperfusion injury (IRI). On the other hand, its physiological inhibitor, the tissue inhibitor of metalloproteinases-1 (TIMP-1), is available in insufficient levels to hamper MMP-9 activity during hepatic IRI. In this study, we generated recombinant adeno-associated virus type 8 vectors (rAAV8) encoding mouse TIMP-1 driven by a liverspecific thyroxine-binding globulin promoter as a strategy to increase the levels of TIMP-1 during liver IRI. Biodistribution analysis confirmed selective overexpression of TIMP-1 in livers of rAAV8 -TIMP-1 vector treated C57BL/6 mice. rAAV8-TIMP-1-treated mice showed reduced MMP-9 activity, diminished leukocyte trafficking and activation, lowered transaminase levels, and improved histology after liver IRI. Moreover, the rAAV8-TIMP-1 vector therapy enhanced significantly the 7day survival rate of TIMP-1-/- mice subjected to hepatic IRI. Neutrophils are the first cells recruited to inflamed tissues and, once activated, they release nuclear DNA-forming web-like structures, known as neutrophil extracellular traps. It was found that TIMP-1 has the ability to reduce formation of neutrophil extracellular traps and, consequently, limit the impact of neutrophil extracellular trap-mediated cytotoxicity in hepatic IRI. This is the first report demonstrating that TIMP-1 overexpression is hepatoprotective in ischemia and reperfusion injury. Hence, TIMP-1 may represent a promising molecule for drug development to treat liver IRI. (Am J Pathol 2018, 188: 1820-1832; https://doi.org/10.1016/j.ajpath.2018.05.002)
\end{abstract}

Hepatic ischemia and reperfusion injury (IRI) is an unavoidable consequence in clinical settings spanning from surgical procedures to pathologies, where the blood supply to liver is temporarily interrupted. ${ }^{1}$ The restoration of blood flow triggers a wide range of inflammatory mediators and pathways that mediate the pathogenesis of liver IRI. ${ }^{1}$

The matrix metalloproteinases (MMPs) are a family of zinc-dependent proteases with important roles in defining how cells interact with their microenvironments. ${ }^{2,3}$ In addition to extracellular matrix turnover, MMPs proteolytically activate various substrates, such as cytokines and chemokines, and have regulatory functions in inflammation and immunity. ${ }^{2,4}$ Among MMPs, MMP-9 is an inducible gelatinase expressed by leukocytes in acutely damaged livers, ${ }^{5}$ and it has emerged as an important pathogenic mediator in hepatic IRI. ${ }^{2}$ MMP-9 is detected in serum samples of liver transplant patients only minutes after reperfusion. ${ }^{6}$ In 2008, we found that MMP-9 deficiency and anti-MMP-9 antibody therapy hamper leukocyte

Supported by the NIH, National Institute of Allergy and Infectious Diseases grant R01AI057832 (A.J.C.). S.D. received partial fellowship salary support from the American Society of Transplantation.

Disclosures: None declared. 
transmigration across liver vascular barriers and profoundly ameliorate hepatic IRI. ${ }^{7}$ In addition to liver IRI, MMP-9 has been regarded as an important therapeutic target in a wide range of inflammatory conditions, including ischemic stroke, arthritis, and islet transplantation. ${ }^{8-10}$

Tissue inhibitors of metalloproteinases (TIMPs) are a family of endogenous inhibitors that bind MMPs in a 1:1 stoichiometry. ${ }^{11}$ There are four identified homologous members of the TIMP family (TIMP-1 through TIMP-4), which differ in structure, tissue-specific expression, and distinct MMP inhibitory activities. ${ }^{11}$ Growing evidence suggests that alterations in MMP-TIMP balances have central roles in the pathogenesis of various diseases. ${ }^{11,12}$ The MMP-9-TIMP-1 balance is of particular interest in hepatic IRI. Indeed, the inability of $T I M P 1^{-1-}$ mice to express TIMP-1 leads to substantially increased MMP-9 activity, severe liver damage, and lethal hepatic IRI. ${ }^{13}$ TIMP-1 has high affinity for MMP-9, ${ }^{14-16}$ and it is more limited in its inhibitory range than other TIMPs. ${ }^{17}$ TIMP-1 is a relatively weak inhibitor against membrane-type MMPs, such as membrane-type 1 MMP (MMP-14), membrane-type 3 MMP (MMP-16), and membrane-type 5 MMP (MMP-24). ${ }^{17}$ Also, the C-terminal domain of TIMP-1, which is important for complex formation with proenzymes, binds more preferentially to the hemopexin domain of MMP-9 than it does to the hemopexin domain of MMP-2, the other member of the gelatinase family. ${ }^{12,16,18}$ In contrast to MMP-9, MMP-2 is constitutively expressed in liver and its loss results in exacerbated liver damage after IRI. $^{3}$

We hypothesized that therapeutic approaches aimed at increasing the levels of TIMP-1 in liver may ameliorate hepatic IRI. To test this concept, recombinant adenoassociated virus (rAAV) serotype 8 vectors, including cloned TIMP-1 cDNA, were generated and packaged into the virus. The AAV vector system is based on a nonpathogenic and replication-defective virus, ${ }^{19,20}$ and it successfully establishes efficient gene expression in vivo without significant toxicity or immune response. ${ }^{21,22}$

\section{Materials and Methods}

\section{Mice and Model of Hepatic IRI}

C57BL/6 mice and C57BL/6 mice carrying the Timp1 null allele (B6.129S4-Timpltm1Pds/J) were obtained directly from either The Jackson Laboratory (Bar Harbor, ME) or its derived breeders housed at University of California, Los Angeles animal facilities under specific pathogen-free conditions. We used a well-established mouse model of warm hepatic IRI. ${ }^{7}$ Briefly, the arterial and portal venous blood supplies were interrupted to the cephalad lobes (median and left lobes) of the liver, which account for $70 \%$ of the liver volume, using an atraumatic clip. After 90 minutes of ischemia, the clip was removed and mice were euthanized after different reperfusion time intervals. In a separate set of experiments, mice were followed up for survival during 7 days after surgery. All animals received humane care, according to the criteria outlined in the Guide for the Care and Use of Laboratory Animals, ${ }^{23}$ published by the NIH. The University of California, Los Angeles Institutional Animal Care and Use Committee approved all study protocols.

\section{Vector Production and Administration}

Recombinant adeno-associated viral TIMP-1 and control vectors were generated according to standard cloning protocols. ${ }^{24}$ Mouse TIMP-1 cDNA was obtained in a nonexpression pSPORT1 vector (Open Biosystems, Lafayette, CO; catalog number MMM1013-202805736; TIMP-1 cDNA accession number BC051260.1). Mouse TIMP-1, green fluorescent protein (GFP), or firefly Luciferase cDNAs were subcloned into AAV cis-plasmids containing the liverspecific thyroxine-binding globulin promoter, the woodchuck hepatitis virus post-transcriptional regulatory element, a polyadenylation signal, and flanking terminal repeats from AAV serotype 2 . Vectors were packaged into rAAV serotype 8 (rAAV8) by the triple transfection method, purified by cesium chloride centrifugation, and titered by quantitative PCR. Large-scale preparations of the viral vectors were

Table 1 List of Primers Used in This Study

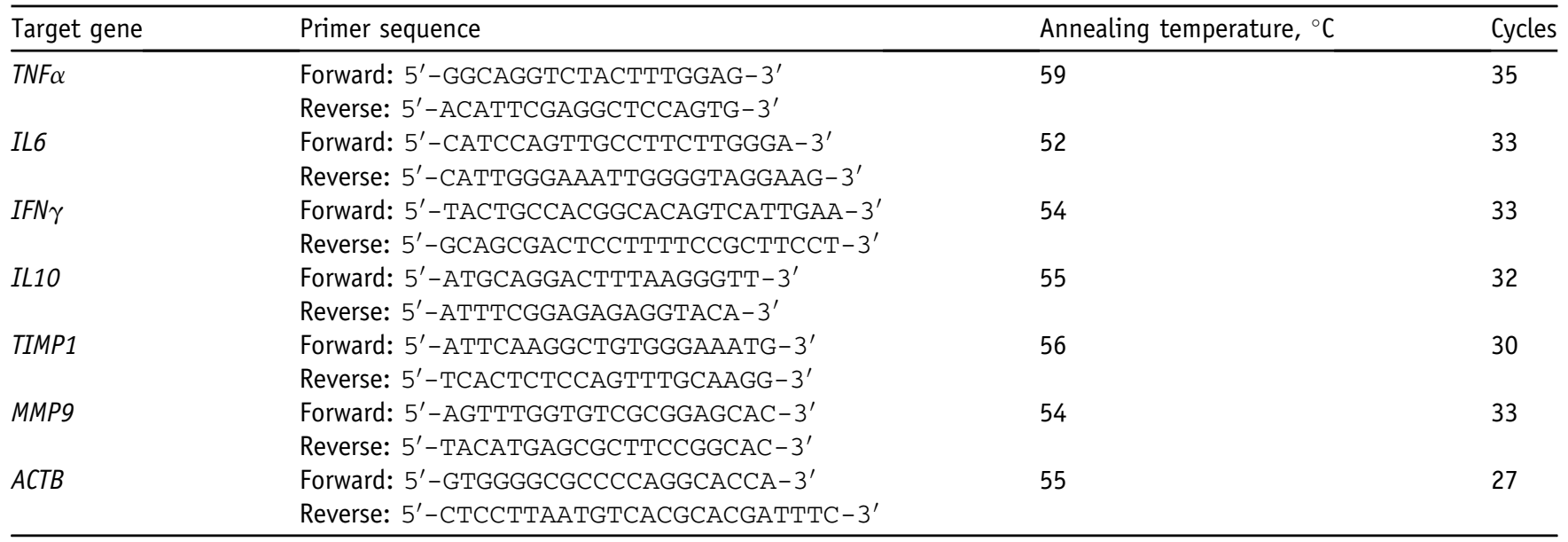




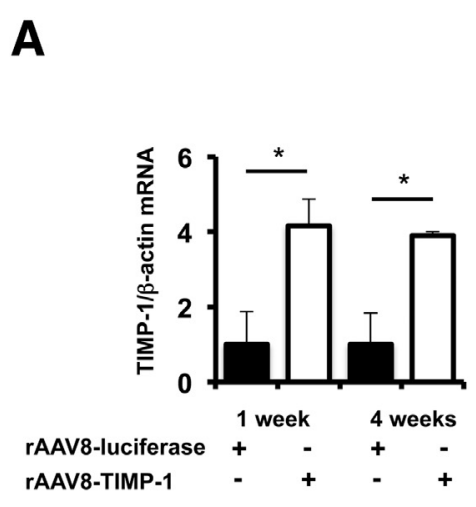

D
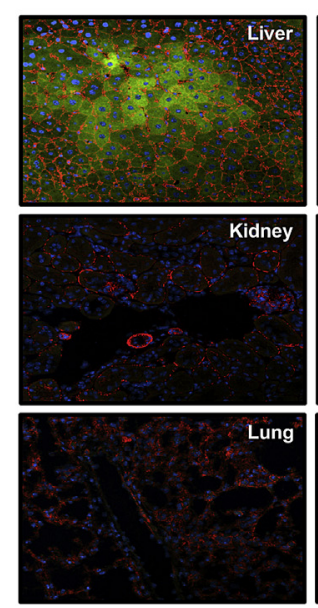

B

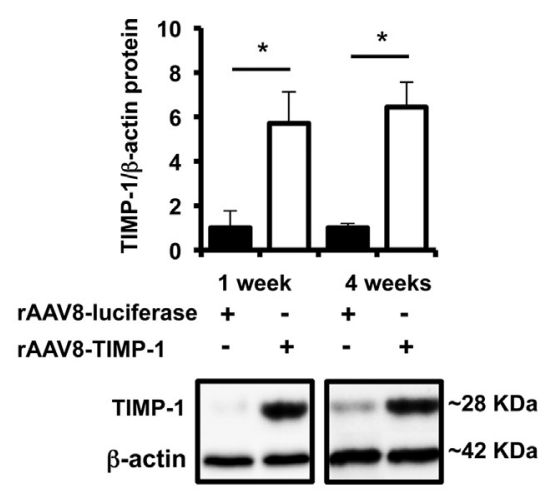

E
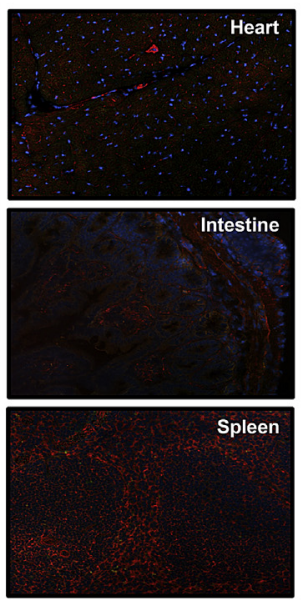

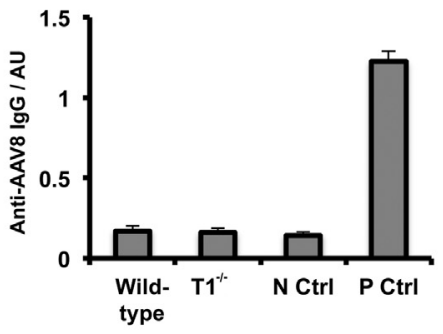

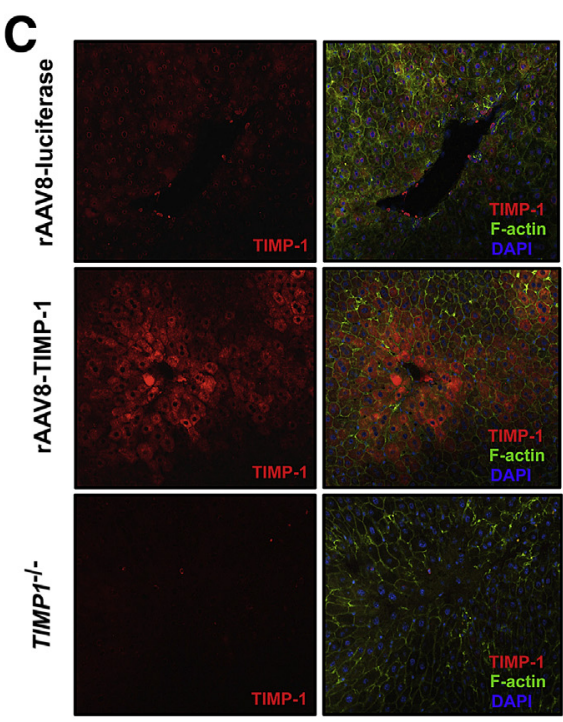

$\mathbf{F}$

Figure 1 Tissue inhibitor of metalloproteinases-1 (TIMP-1) biodistribution before hepatic ischemia and reperfusion injury. A and B: TIMP1 mRNA (A) and TIMP-1 protein (B) were overexpressed in livers 1 and 4 weeks after recombinant adeno-associated virus type 8 (rAAV8)-TIMP-1 vector administration. Black bars indicate controls; and white bars, rAAV8-TIMP-1-treated livers. C: TIMP-1 deposition in the perivascular areas is scarce in rAAV8-luciferase and elevated in rAAV8-TIMP1 -transduced livers 1 week after vector injection. TIMP1-/- livers are negative for TIMP-1 staining; TIMP-1 is shown in red (Alexa Fluor 594), F-actin in green (Phalloidin Alexa Fluor 488), and nuclear DAPI stain in blue. D: Green fluorescent protein (GFP) fluorescence in mice 1 week after rAAV8-GFP vector injection confirms that GFP expression (under the control of the thyroxine-binding globulin promoter) is detected in liver and absent from kidney, lung, heart, intestine, and spleen. GFP is shown in green, F-actin in red (Phalloidin Alexa Fluor 594), and nuclear DAPI stain in blue. E and F: Mice have absence of anti-AAV8 capsid humoral immunity before rAAV8 administration (E) and show virtually no histologic damage after rAAV8 injection (F). Data are expressed as means \pm SEM. $n=4$ to 5 per group (A-F). ${ }^{*} P<0.05$. Original magnification: $\times 20(\mathbf{C}$ and $\mathbf{D}) ; \times 10(\mathbf{F})$. AU, arbitrary unit; $\mathrm{N}$ Ctrl, negative control; $\mathrm{P}$ Ctrl, positive control; $\mathrm{T}^{-/-}, \mathrm{TIMP} 1^{-/-}$.

custom ordered from the Penn Vector Core (University of Pennsylvania, Philadelphia, PA). rAAV8-TIMP-1, rAAV8GFP, and rAAV8-luciferase vectors were diluted in pharmaceutical grade saline and administered $\left(5 \times 10^{12}\right.$ genome copies per kilogram, intravenously) to mice 1 and 4 weeks before euthanasia for in vivo biodistribution analysis and 1 week before surgery for hepatic IRI studies, including the 7-day mouse survival study.

\section{AAV8 Antibody Detection in Mouse Serum}

Previous exposure to AAV8 viral capsid was assessed in mouse serum samples by enzyme-linked immunosorbent assay. ${ }^{25}$ Enzyme-linked immunosorbent assay plates were coated with $1 \times 10^{9}$ genome copies AAV8 per well. Positive control serum samples were collected from AAV8 injected mice with established anti-AAV8 antibody titers.

\section{Assessment of Liver Damage}

Liver specimens were fixed in $10 \%$ buffered formalin, embedded in paraffin, and processed for hematoxylin and eosin and Sirius Red staining, as previously described. ${ }^{26}$ Serum alanine transaminase and aspartate transaminase levels were evaluated using a commercial kit (Teco Diagnostics, Anaheim, CA). 
A

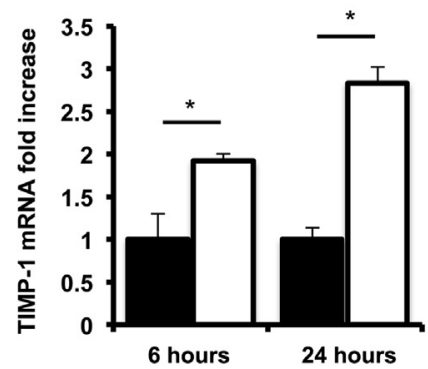

rAAV8-TIMP-1 $-\quad+\quad-\quad+$

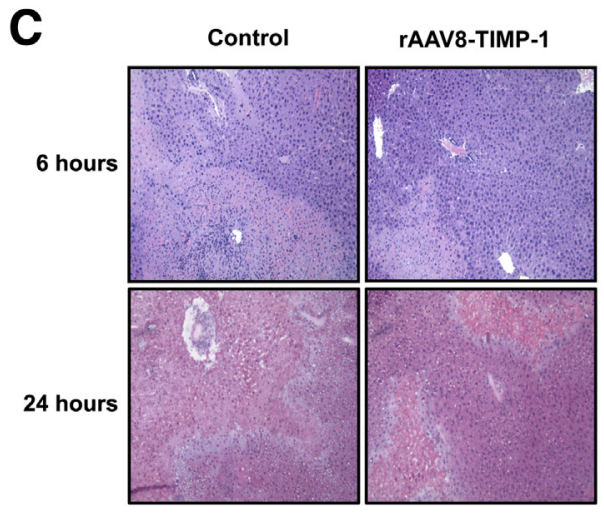

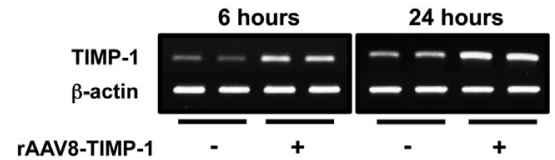

D

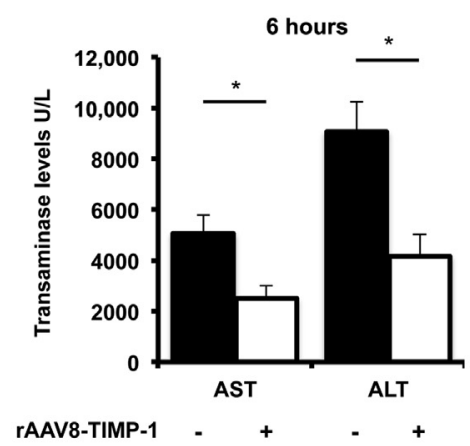

B
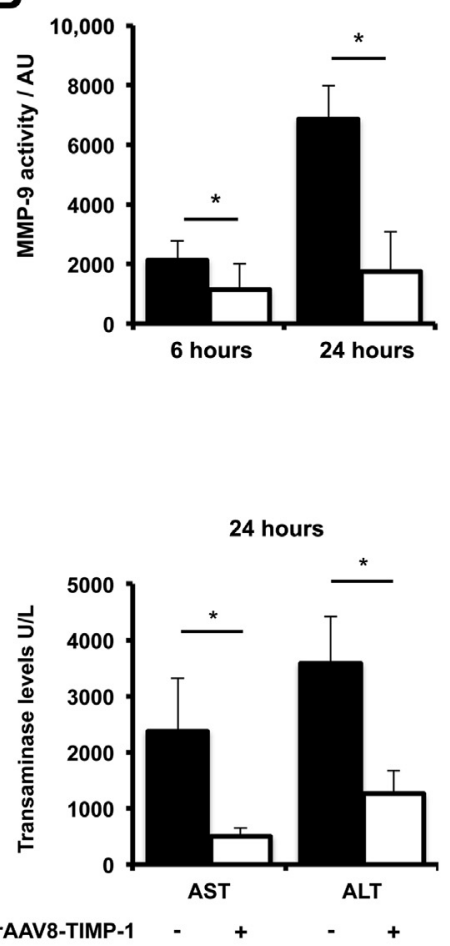

Figure 2 Tissue inhibitor of metalloproteinases-1 (TIMP-1) expression, matrix metalloproteinase (MMP)-9 activity, histology, and transaminase levels after liver ischemia and reperfusion injury (IRI). A and B: TIMP-1 is significantly overexpressed in recombinant adeno-associated virus type 8 (rAAV8)-TIMP1-treated livers (A) and correlates with a profound depression in MMP-9 activity in these livers at 6 and 24 hours after IRI (B). C: Hematoxylin and eosin staining shows that compared with controls, rAAV8-TIMP-1-treated livers have reduced lobular architecture disruption and hepatocellular injury at 6 and 24 hours after IRI. D: Serum aspartate transaminase (AST) and serum alanine transaminase (ALT) levels are significantly lower in rAAV8-TIMP-1-treated mice after IRI. Closed bars indicate controls; and open bars, rAAV8-TIMP-1-treated livers. Data are expressed as means \pm SEM. $n=6$ per group $\left(\right.$ A - D). ${ }^{*} P<0.05$. Original magnification, $\times 10$. $\mathrm{AU}$, arbitrary unit.

\section{Isolation and Culture of Mouse Cells}

Isolation of neutrophils and hepatocytes was performed according to previously published methods. ${ }^{3,27}$ Briefly, to isolate neutrophils, bone marrow was flushed from femurs and tibias (stripped of all muscle and sinew) with $2.5 \mathrm{~mL}$ of RPMI-1640 medium containing 5\% fetal calf serum on ice. Hypotonic lysis was used to remove erythrocytes. The bone marrow flush was resuspended in Hanks' balanced saline solution, layered on a Percoll (Sigma-Aldrich, St. Louis, MO) gradient (3 $\mathrm{mL}$ of $55 \%$, top; $3 \mathrm{~mL}$ of $65 \%$, middle; $4 \mathrm{~mL}$ of $80 \%$ Percoll), and centrifuged at $872 \times g$ for 30 minutes at $10^{\circ} \mathrm{C}$. Mature neutrophils were collected from the interface of the $65 \%$ and $80 \%$ fractions. The neutrophil-rich fractions were $>90 \%$ pure (Ly-6G immunostaining/morphology) and $>95 \%$ viable (trypan blue exclusion). To isolate hepatocytes, anesthetized mice were subject to a midline laparotomy and cannulation of the inferior vena cava for liver perfusion with EDTA chelating and collagenase perfusion buffers. Hepatocytes were separated from nonparenchymal cells by successive low-speed centrifugation steps and resuspended in Williams E Medium with primary hepatocyte maintenance supplements (Life Technologies, Carlsbad, CA).

\section{Immunoperoxidase and Immunofluorescence Assays}

Acetone-fixed cryostat liver sections or $4 \%$ paraformaldehyde fixed neutrophils were incubated with anti-Ly-6G (1A8; BD Biosciences, San Jose, CA), antimacrophage-1 antigen (Mac-1 M1/70; BD Biosciences), anti-MMP-9 (AF909; R\&D Systems, Minneapolis, MN), anti-TIMP-1 (AF980; R\&D Systems), and anti-citrullinated histone H3 (Cit-H3; Abcam, Cambridge, MA) antibodies, as described. ${ }^{13}$ The sections were evaluated blindly (S.D.) by counting 10 highpower fields/section in triplicate. Dual/triple staining was detected by immunofluorescence with Alexa Fluor 594-red anti-rabbit IgG and anti-goat IgG $(\mathrm{H}+\mathrm{L})$, Alexa Fluor 488green anti-rat $\operatorname{IgG}(\mathrm{H}+\mathrm{L})$, Alexa Fluor 350-red anti-rabbit $\operatorname{IgG}(\mathrm{H}+\mathrm{L})$, and Alexa Fluor 594 Phalloidin (Thermo Fisher Scientific, Waltham, MA). Slides were analyzed under a Nikon Eclipse 90i Fluorescence Microscope (Nikon, Melville, NY).

\section{Gelatin Zymography Analysis}

Liver protein extraction and zymography analyses were performed as previously described. ${ }^{7}$ Gelatinolytic activity was detected in liver extracts (100 $\mu \mathrm{g}$ final protein content) using $10 \%$ SDS-PAGE gels containing $1 \mathrm{mg} / \mathrm{mL}$ of gelatin 


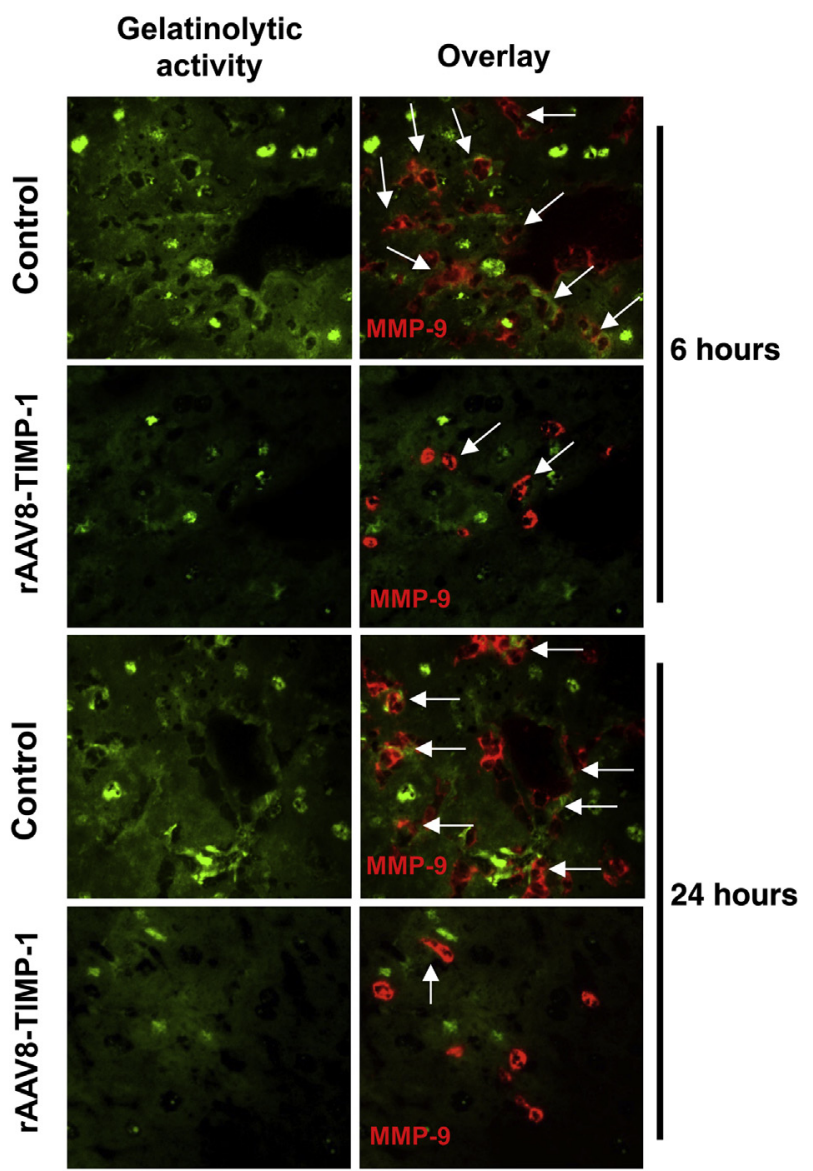

Figure 3 Gelatinolytic activity assessed by in situ zymography in hepatic ischemia and reperfusion injury (IRI). The green fluorescence caused by the breakdown of DQ-gelatin is largely reduced in the recombinant adeno-associated virus type 8 (rAAV8)-tissue inhibitor of metalloproteinases-1 (TIMP-1)-treated livers after 6 and 24 hours of hepatic IRI when compared with controls. Combined immunofluorescence staining of matrix metalloproteinase (MMP)-9 (red) and gelatinolytic activity (green) in the same cryostat sections reveals relatively low MMP-9 gelatinolytic activity in rAAV8-TIMP-1-treated livers after IRI (the arrows indicate gelatinolytic activity in MMP- $9^{+}$cells). $n=5$ to 6 per group. Original magnification, $\times 40$.

(Thermo Fisher Scientific) under nonreducing conditions. Recombinant MMPs (BIOMOL International, Plymouth, PA) and prestained molecular weight markers (Bio-Rad Laboratories, Hercules, CA) served as standards.

\section{In Situ Zymography}

Cellular localization of gelatinolytic activity was examined on frozen and unfixed liver cryosections by in situ zymography, as previously described. ${ }^{28}$ First, MMP-9 protein was immunofluorescently stained with goat anti-MMP-9 (AF909; R\&D Systems) primary antibody and an Alexa Fluor 594 anti-goat IgG $(\mathrm{H}+\mathrm{L})$ (Thermo Fisher Scientific). Subsequently, quenched fluorogenic DQ-gelatin substrate (Thermo Fisher Scientific) was dissolved at $1 \mathrm{mg} / \mathrm{mL}$ in zymogram developing buffer (diluted, 1:10; Thermo Fisher
Scientific) and then diluted 1:10 in Agarose type VII-A, low gelling temperature $(1 \% \mathrm{w} / \mathrm{v}$; Sigma-Aldrich). Fifty microliters of DQ-gelatin solution in agarose was overlayed on each liver cryosection, and slides were incubated for 4 hours at $37^{\circ} \mathrm{C}$ before gelatinolytic activity was detected by fluorescence microscopy with the use of a fluorescein isothiocyanate filter. Experimental controls included liver sections overlayed with just agarose or DQ-gelatin/agarose containing $20 \mathrm{mmol} / \mathrm{L}$ EDTA (Sigma-Aldrich).

\section{Immunoblotting}

Western blots were performed with protein lysates from liver tissue, isolated hepatocytes, and neutrophils, and concentrated cell culture medium from in vitro assays. ${ }^{13}$ Lysates (40 $\mu \mathrm{g} / \mathrm{well})$ were separated in $10 \%$ to $15 \%$ SDSPAGE gels and transferred to polyvinylidene difluoride membranes, which were incubated overnight with primary antibodies against TIMP-1 (1:500; R\&D Systems), Cit-H3 (1:1000; Abcam), GFP (1:1000; Santa Cruz Biotechnology, Dallas, TX), and $\beta$-actin (1:16,000; Abcam) as an internal control for each membrane.

\section{RNA Extraction and RT-PCR}

RNA was extracted with Trizol (Thermo Fisher Scientific), as previously described. ${ }^{7}$ Reverse transcription of 0.5 to 5 $\mu \mathrm{g}$ of total RNA was performed with the SuperScript III first-strand cDNA synthesis supermix (Thermo Fisher Scientific). The cDNA products were amplified by PCR using primers specific for each target cDNA (Table 1).

\section{Quantification of NETs and Nuclear Expansion in Vitro}

Nuclear area expansion and neutrophil extracellular trap (NET) formation were quantified, as previously described. ${ }^{29}$ Briefly, freshly isolated neutrophils $\left(3 \times 10^{5} /\right.$ well $)$ were seeded in 24-well plates in RPMI 1640 media and plated for 60 minutes at $37^{\circ} \mathrm{C}$ for cellular adhesion to the uncoated plates. Neutrophils were then stimulated for 3 hours with either $100 \mathrm{nmol} / \mathrm{L}$ of phorbol 12-myristate 13-acetate (PMA) or variable concentrations of media collected from primary hepatocytes cultured under hypoxic conditions $(1 \%$ $\mathrm{O}_{2}$ ). Wherever indicated, cells were either pretreated with $200 \mu \mathrm{mol} / \mathrm{L}$ of protein arginine deiminase 4 inhibitor, $\mathrm{Cl}$ amidine (Caymen Chemical, Ann Arbor, MI) for 30 minutes before stimulation or stimulated in the presence of recombinant mouse TIMP-1 (rTIMP-1; Biolegend, San Diego, CA). Sytox green, at a final concentration of 160 $\mathrm{nmol} / \mathrm{L}$ (Thermo Fisher Scientific), was added to each well and incubated in the dark for 10 minutes before visualization of NETs by fluorescence microscopy. Fluorescence and phase-contrast images were taken per well on an Olympus IX7I inverted microscope (Olympus IMS, Waltham, MA). Using ImageJ64 image software version 1.47 (NIH, Bethesda, MD; http://imagej.nih.gov/ij), the area of each 
A

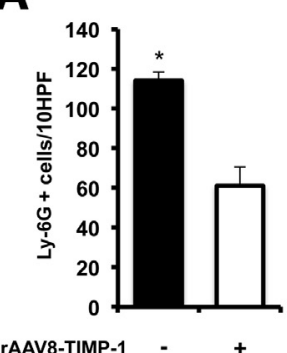

B

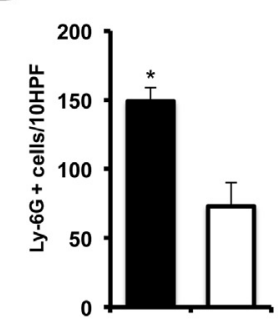

IAAV8-TIMP-1 - +

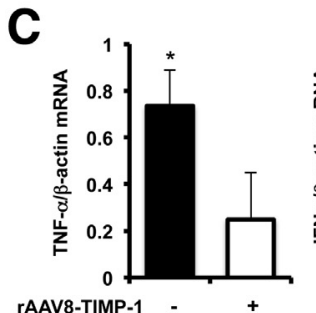

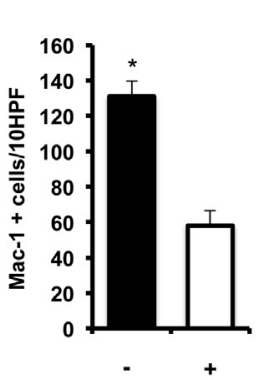
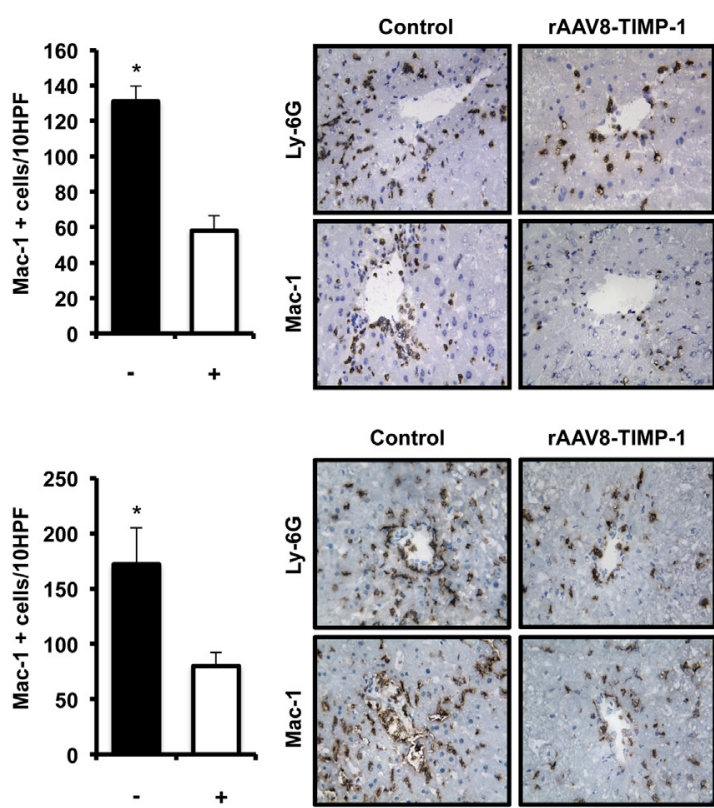

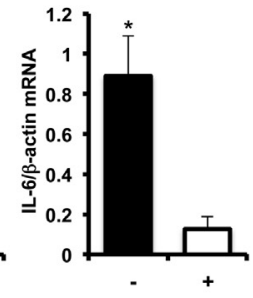

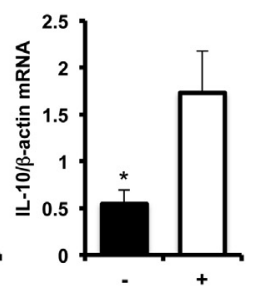

Figure 4 Leukocyte infiltration and cytokine expression after liver ischemia and reperfusion injury (IRI). A and B: Livers harvested at 6 hours (A) and 24 hours (B) after IRI show that recombinant adeno-associated virus type 8 (rAAV8)-tissue inhibitor of metalloproteinases-1 (TIMP-1) vector-treated livers have significantly reduced $\mathrm{Ly}-6 \mathrm{G}^{+}$ neutrophil and Mac- $1^{+}$leukocyte infiltration, compared with respective controls. C: Tumor necrosis factor (TNF)- $\alpha$, interferon (IFN) $-\gamma$, and IL- 6 are markedly depressed and IL-10 is significantly up-regulated in rAAV8-TIMP-1 vector-treated livers at 6 hours after IRI. Closed bars indicate controls; and open bars, rAAV8-TIMP-1-treated livers. Data are expressed as means \pm SEM. $n=4$ to 6 mice per group $(\mathbf{A}-\mathbf{C})$. ${ }^{*} P<0.05$. Original magnification, $\times 40$. $\mathrm{HPF}$, high-power field.
Sytox signal (nuclear expansion area) in the fluorescent image was individually measured. The percentage of Sytox-positive cells was obtained by dividing the Sytoxpositive cell counts by the total number of cells (250 to 500 cells/image) in the corresponding phase-contrast images. The distribution of the number of cells across the range of nuclear areas was obtained using the frequency function in Excel 2011 version 14.1.0. (Microsoft, Redmond, WA).

\section{Statistical Analysis}

Results are presented as means \pm SEM. The statistical analyses were performed using the unpaired $t$-test to compare different groups. The statistical significance of differences in mouse survival was determined by Kaplan-Meier analysis. Statistical significance was assigned at $P<0.05$.

\section{Results}

\section{Efficient Hepatic TIMP-1 Transduction after rAAV8-TIMP-1 Vector Injection}

To achieve constitutive high-level expression of TIMP-1 in livers, AAV serotype 8 vectors, including cloned TIMP-1 cDNA, were generated and packaged into the virus. The
rAAV8-TIMP-1 vectors were under the control of the liver-specific thyroxine-binding globulin gene promoter. ${ }^{30}$ Biodistribution studies demonstrated TIMP1 mRNA and protein overexpression in livers as early as 1 week after rAAV8-TIMP-1 vector injection, compared with mice treated with control rAAV8-Luciferase or rAAV8-GFP viruses (Figure 1, A and B). TIMP-1 protein overexpression was mostly detected in perivascular areas (Figure 1C). Detection of GFP fluorescence in mice treated with rAAV8GFP vectors confirmed that the thyroxine-binding globulin promoter was actively driving expression in livers and virtually silenced in the other evaluated organs (Figure 1D). Mice used in this study were naïve to AAV8 exposure before rAAV8 vector administration (Figure 1E), and rAAV8driven TIMP-1 and GFP/luciferase expressions were achieved without noticeable hepatic inflammation or cell death (Figure 1F).

\section{AAV-Mediated TIMP-1 Overexpression Attenuates Hepatic IRI}

The rAAV8-TIMP-1 vectors were administered to C57B/6 mice 1 week before IRI; control mice received an identical dose of rAAV8-GFP or saline. No statistical differences were observed on TIMP-1 expression, MMP-9 activity, serum transaminases, and liver histology between rAAV8-GFP vector- and 
A
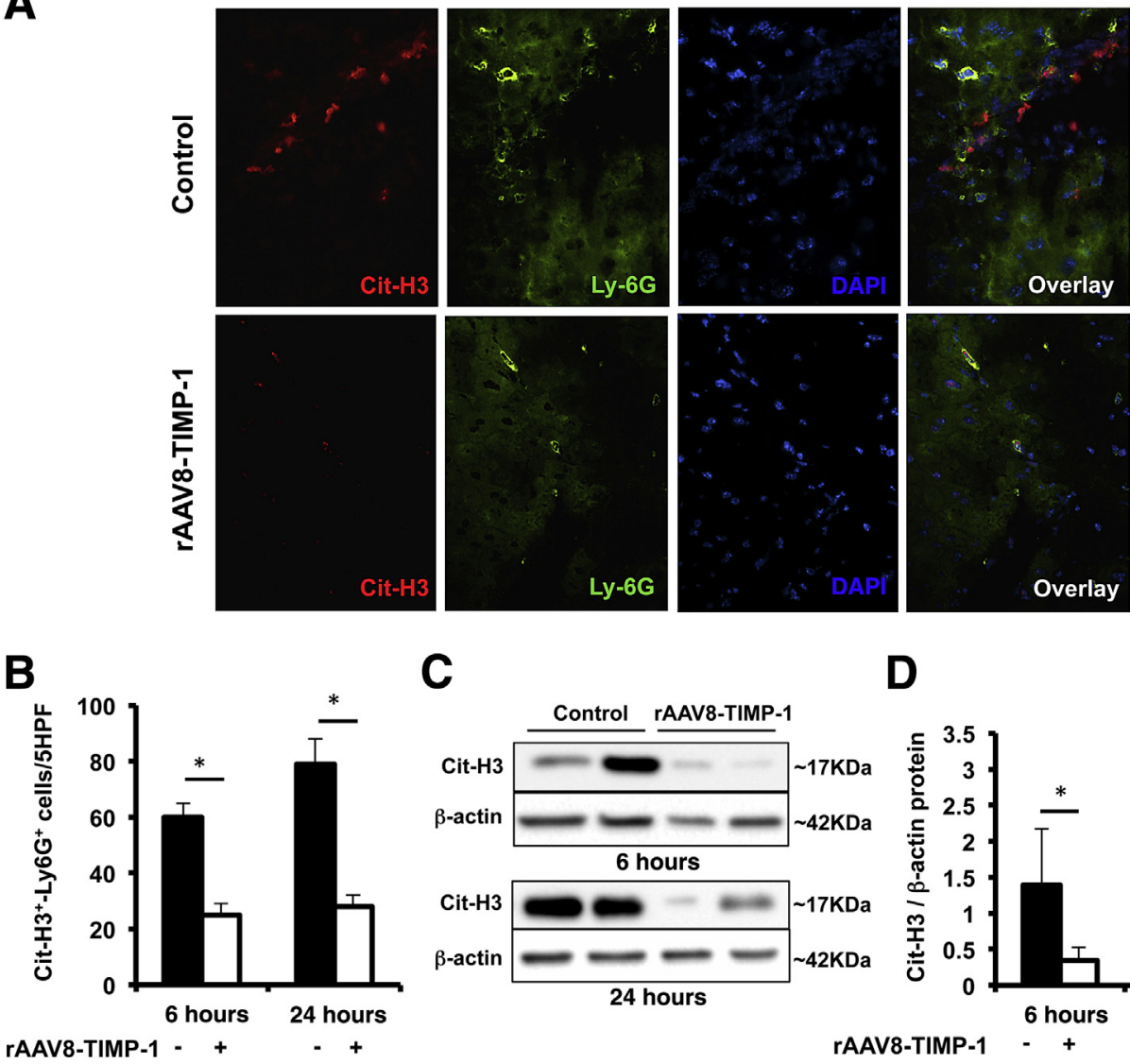

C

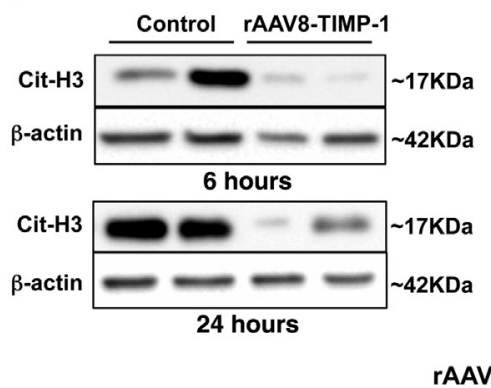

D

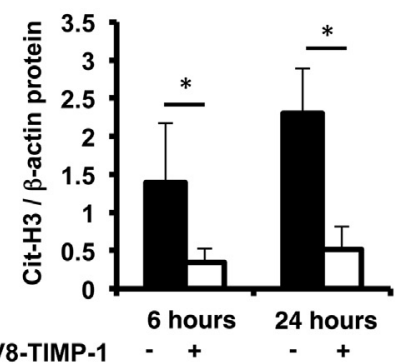

$\mathbf{E}$
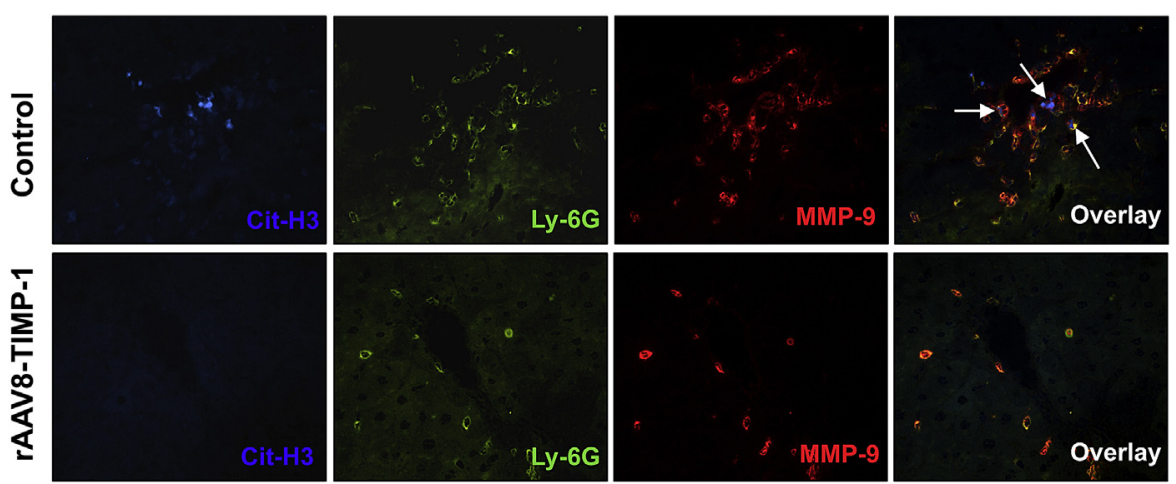

Figure 5 Adeno-associated virus type 8 (AAV8)-mediated transduction of tissue inhibitor of metalloproteinases-1 (TIMP-1) reduces neutrophil extracellular trap (NET) formation in hepatic ischemia and reperfusion injury (IRI). A: NET formation assessed by triple immunofluorescence staining in control and recombinant AAV8 (rAAV8)-TIMP-1-treated livers at 6 hours (6h) after IRI; citrullinated histone H3 (Cit-H3) in red (Alexa Fluor 594), Ly-6G in green (Alexa Fluor 488), and nuclear DNA DAPI stain in blue. B: The number of Cit-H3 ${ }^{+}$cells associated with Ly-6G antigen is significantly depressed in rAAV8-TIMP1 -treated livers at both 6 and 24 hours (24 h) after IRI. C and D: Western blot analysis of citrullinated histone H3 (C) and Cit-H3/ $\beta$-actin ratios (D) reveals a significant decrease in $\mathrm{H} 3$ citrullination in the ischemic lobes of rAAV8-TIMP-1-treated livers at 6 and 24 hours after reperfusion. E: NET-associated matrix metalloproteinase (MMP)-9 assessed by triple immunofluorescence staining in control and rAAV8-TIMP-1-treated livers at 6 hours after IRI; Cit-H3 in blue (Alexa Fluor 350), Ly-6G in green (Alexa Fluor 488), and MMP-9 in red (Alexa Fluor 594). Closed bars indicate controls; and open bars, rAAV8-TIMP-1-treated livers. The arrows indicate colocalization of MMP-9 and citrullinated histone $\mathrm{H} 3$ in Ly- $6 \mathrm{G}^{+}$neutrophils. Data are expressed as means \pm SEM. $n=5$ to 6 mice per group $(\mathbf{A}-\mathbf{E}) .{ }^{*} P<0.05$. Original magnification: $\times 20(\mathbf{A}) ; \times 40(\mathbf{E}) . \mathrm{HPF}$, high-power field.

vehicle-treated controls. TIMP1 mRNA expression was significantly increased in rAAV8-TIMP-1-treated mice at both 6 and 24 hours after IRI, compared with controls (Figure 2A). The elevated levels of TIMP-1 expression in the rAAV8-TIMP1 -treated livers correlated with a significant reduction in
MMP-9 activity evaluated by gelatin zymography (Figure 2B). Overall, TIMP-1-overexpressing livers had reduced hepatocyte necrosis, sinusoidal congestion, and moderate edema at both 6 and 24 hours after IRI (Figure 2C), and improved function, as evidenced by the reduced serum transaminase levels after IRI 
A

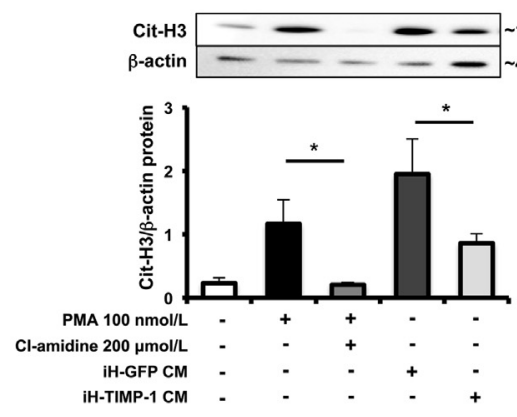

D
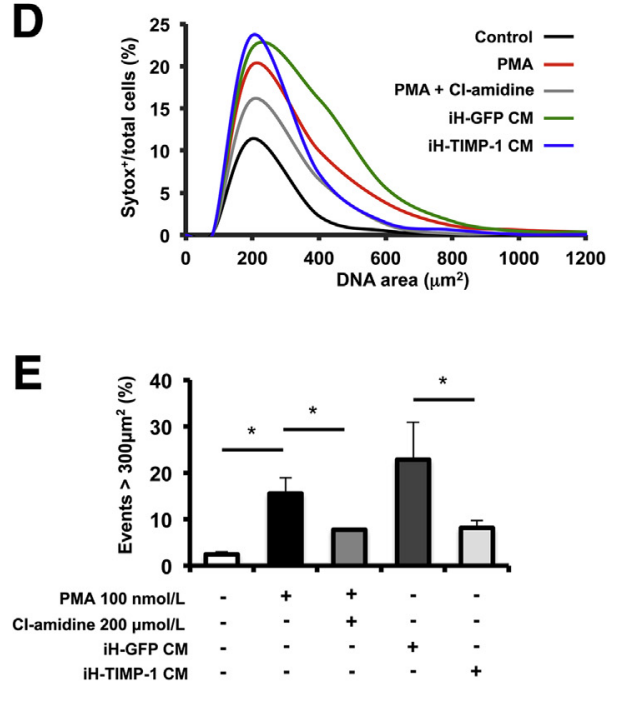

B

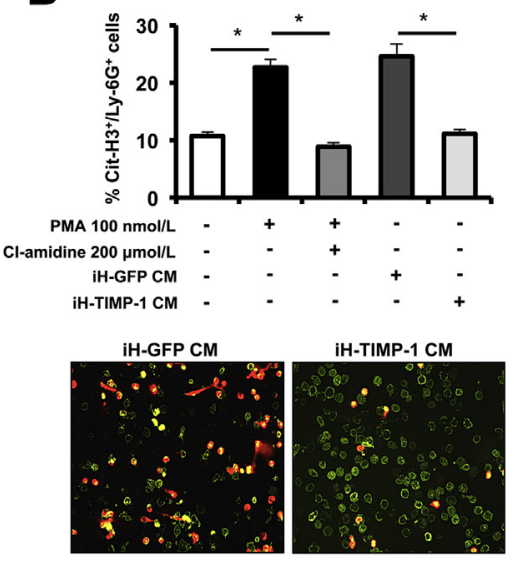

C

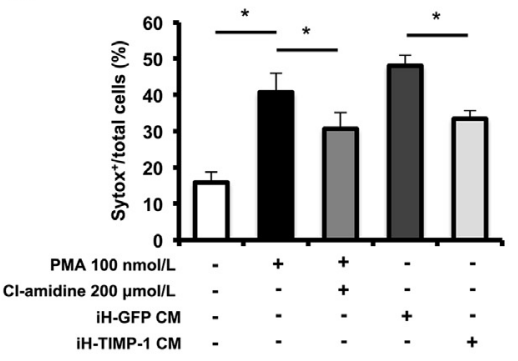

Figure 6 Tissue inhibitor of metalloproteinases1 (TIMP-1)-rich hepatocyte conditioned medium (iH-TIMP-1) hampers neutrophil extracellular trap formation. A and B: Citrullinated histone $\mathrm{H} 3$ (Cit-H3) protein levels (A) and the percentage of $\mathrm{Cit}^{-} \mathrm{H}^{+}{ }^{+}$cells associated with Ly6G antigen (B) are both significantly depressed in isolated bone marrow neutrophils treated with iH-TIMP-1 CM, compared with neutrophils treated with conditioned media obtained from GFP-expressing primary hepatocytes (iH-GFP CM); Cit-H3 stained in red (Alexa 594), and Ly-6G stained in green (Alexa Fluor 488). Neutrophils stimulated with PMA in the absence or presence of $\mathrm{Cl}$-amidine serve as positive and negative controls, respectively. C: The percentage of Sytox ${ }^{+}$ events is significantly reduced in neutrophil cultures treated with either $\mathrm{iH}-\mathrm{TIMP}-1 \mathrm{CM}$ or $\mathrm{Cl}$-amidine in the presence of Sytox green, the cell-impermeable DNA dye. D: Distribution plot of the percentage of Sytox ${ }^{+}$events (y axis) across the range of chromatin decondensation (Sytox stain) area size ( $x$ axis). E: The percentage of Sytox ${ }^{+}$neutrophils with extracellular DNA areas $>300 \mu \mathrm{m}^{2}$ [neutrophil extracellular trap (NET) formation] is markedly depressed in neutrophils treated with iH-TIMP-1 CM. F: Representative Sytox green extracellular DNA fluorescence and phasecontrast images of neutrophils used for NET quantification. White bars indicate untreated; black bars, PMA stimulated; medium gray bars, PMA stimulated in the presence of $\mathrm{Cl}$-amidine; dark gray bars, iH-GFP CM stimulated; and light gray bars, $\mathrm{iH}-\mathrm{TIMP}-1 \mathrm{CM}$-treated neutrophils. In vitro data are expressed as means \pm SEM of four independent experiments $(\mathbf{A}-\mathbf{C}$ and $\mathbf{E}) .{ }^{*} P<0.05$. Original magnification: $\times 40(B) ; \times 20($ F). PMA, phorbol 12-myristate 13 -acetate.
(Figure 2D). The detection of enzymatic activity in cryostat sections by in situ zymography confirmed a significantly reduced gelatinolytic activity in the RAAV8-TIMP-1-treated livers after IRI; the green fluorescence attributable to DQ-gelatin breakdown (gelatinolytic activity) was abundantly detected in control sections, and it was markedly reduced in the TIMP1-overexpressing livers after hepatic IRI (Figure 3). Hence, enhanced levels of TIMP-1 clearly mitigated gelatinolytic activity and attenuated hepatic IRI.

\section{AAV-Mediated TIMP-1 Overexpression Reduces Leukocyte Infiltration and Activation in Hepatic IRI}

The migration of $\mathrm{Ly}-6 \mathrm{G}^{+}$neutrophils and Mac- $1^{+}$leukocytes was significantly depressed in rAAV8-TIMP1-treated livers after hepatic IRI (Figure 4, A and B), which correlated with the reduced numbers of MMP- $9^{+}$leukocytes detected in these livers (Supplemental Figure S1).
Infiltrating leukocytes are the sources of MMP-9 in acutely damaged livers. ${ }^{7}$ TIMP-1 overexpression resulted in downregulation of inflammatory cytokines (tumor necrosis factor$\alpha$, interferon- $\gamma$, and IL-6) and up-regulation of the anti-inflammatory IL-10 after liver IRI (Figure 4C).

\section{TIMP-1 Protein 0verexpression Hampers the Formation of Neutrophil Extracellular Traps after Hepatic IRI}

Neutrophils are recruited early to inflamed tissues and are capable of damaging any tissue in the body. ${ }^{31}$ They produce extracellular DNA webs called NETs within minutes after activation. ${ }^{32}$ Livers were triple immunofluorescently labeled for Cit-H3, a widely used NET-specific marker, ${ }^{33}$ Ly-6G, and DAPI (unlike Cit-H3, does not stain extracellular DNA). The number of Cit-H3-positive Ly-6G neutrophils was significantly depressed in the rAAV8-TIMP-1 vector-treated livers, compared with controls at 6 and 24 hours after IRI 
A

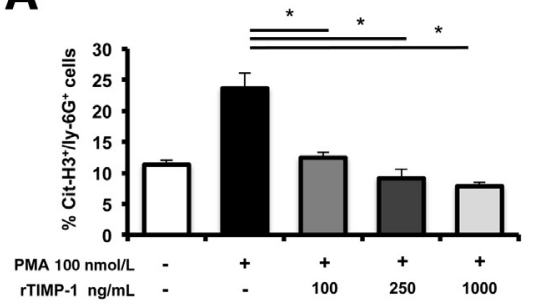

C

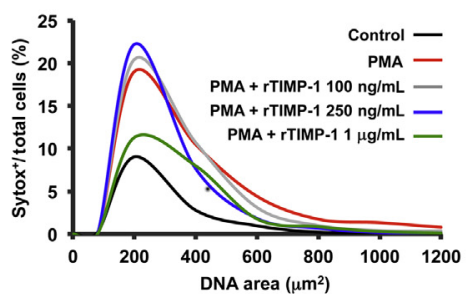

B

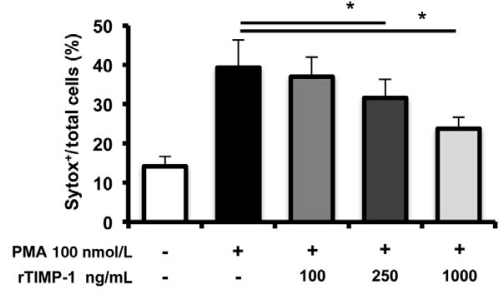

D

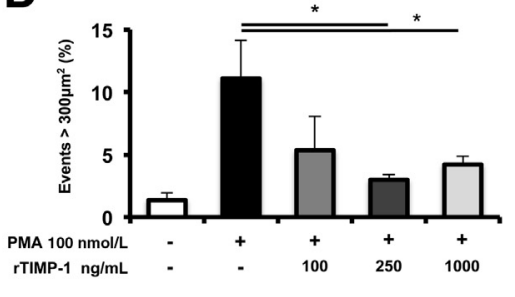

Figure 7 Recombinant tissue inhibitor of metalloproteinases-1 (rTIMP-1) depresses neutrophil extracellular trap (NET) formation in vitro. A and B: The percentage of NET-forming double-positive citrullinated histone $\mathrm{H} 3(\mathrm{Cit}-\mathrm{H} 3)^{+} / \mathrm{Ly}^{-6 \mathrm{G}^{+}}$neutrophils (A) and the percentage of Sytox ${ }^{+}$events (B) are significantly reduced in PMA-stimulated neutrophils treated with mouse rTIMP-1. C: Analysis of the Sytox ${ }^{+}$event (\%; $y$ axis) distribution across the range of neutrophil extracellular chromatin decondensation areas (Sytox stain; $x$ axis). D: The percentage of Sytox ${ }^{+}$neutrophils with extracellular DNA areas $>300 \mu \mathrm{m}^{2}$ is markedly depressed in rTIMP-1-treated PMA-stimulated neutrophils. E: Representative Sytox green extracellular DNA fluorescence and phase-contrast images of neutrophils used for NET quantification. White bars indicate untreated; black bars, PMA stimulated; and gray bars, PMA stimulated in the presence of rTIMP-1. In vitro data are expressed as means \pm SEM of three independent experiments (A, B, and D).

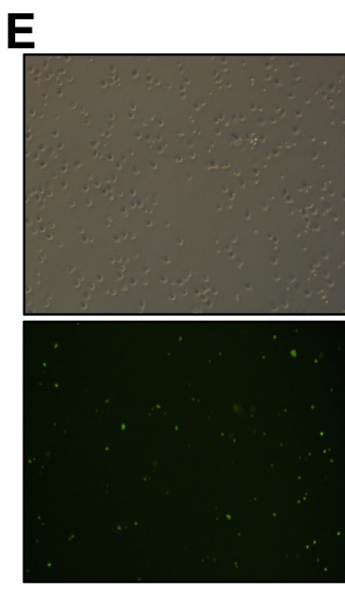

Control

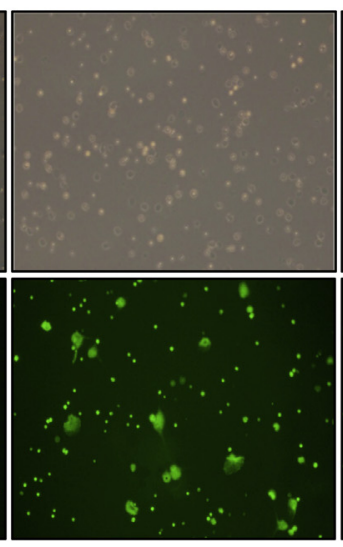

PMA

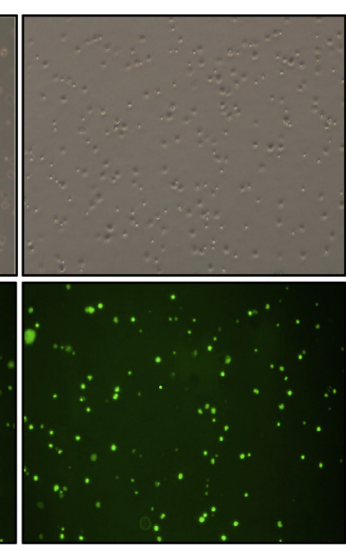

PMA

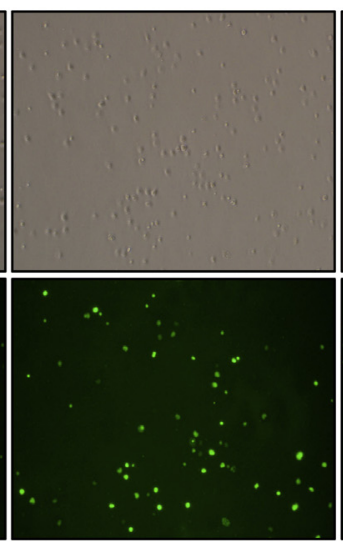

PMA

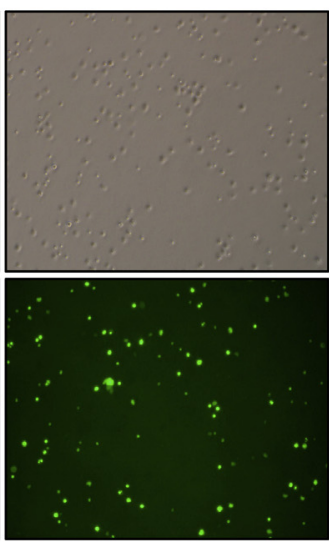

PMA

rTIMP-1 $100 \mathrm{ng} / \mathrm{mL} \quad$ rTIMP-1 $250 \mathrm{ng} / \mathrm{mL} \quad$ rTIMP-1 $1000 \mathrm{ng} / \mathrm{mL}$

(Figure 5, A and B). Western blot analysis for total Cit-H3 levels also confirmed that neutrophil NET formation was impaired in TIMP-1-overexpressing livers after IRI (Figure 5, $\mathrm{C}$ and D). MMP-9 immunofluorescence colocalized with Cit$\mathrm{H} 3$ in $\mathrm{Ly}-6 \mathrm{G}^{+}$neutrophils infiltrating the liver ischemic lobes (Figure 5E). NET-forming neutrophils with NET bound MMP-9 were particularly detected in the hepatic perivascular areas of control livers after IRI. Livers in which MMP-9 activity was depressed by rAAV8-TIMP-1 vector therapy showed reduced MMP-9 and Cit-H3 colocalization in $\mathrm{Ly}-6 \mathrm{G}^{+}$ neutrophils (Figure 5E). Hence, these data suggest that TIMP-1 overexpression affects the ability of neutrophils to form extracellular traps in response to hepatic IRI.

\section{Hepatocyte-Derived TIMP-1 and rTIMP-1 Depress NET Formation in Vitro}

To test the role of TIMP-1 in NET formation without the cell infiltration contributing factor, isolated neutrophils were stimulated with either PMA or conditioned media from AAV-transduced hepatocytes subject to hypoxia. NET formation was strongly induced in PMA-simulated neutrophils, compared with either PMA-simulated neutrophils pretreated with $\mathrm{Cl}$-amidine (protein arginine deiminase 4 inhibitor) or unstimulated neutrophils. NET formation was also profoundly increased in neutrophils cultured with conditioned media obtained from GFP-expressing primary hepatocytes (iH-GFP CM). However, compared with iH-GFP CM treated controls, the Cit-H3 levels were significantly depressed in neutrophils treated with conditioned media from TIMP-1-overexpressing hepatocytes (iH-TIMP-1 CM) (Figure 6A). Immunofluorescence staining confirmed that PMA and $\mathrm{iH}-\mathrm{GFP} \mathrm{CM}$ strongly increased the number of Cith $3^{+} / \mathrm{Ly}^{-6 \mathrm{G}^{+}}$cells, whereas both $\mathrm{Cl}$-amidine and $\mathrm{iH}-\mathrm{TIMP}-1 \mathrm{CM}$ reduced the number of NET forming cells (Figure 6B). Experiments were repeated in the presence of Sytox green, a cell-impermeable nuclear acid dye used as a marker of chromatin decondensation and NET release. 
Herein, iH-TIMP-1 CM and protein arginine deiminase 4 inhibition significantly reduced the percentage of Sytox ${ }^{+}$ neutrophils when compared with neutrophils cultured in $\mathrm{iH}-$ GFP CM and PMA-stimulated neutrophils, respectively

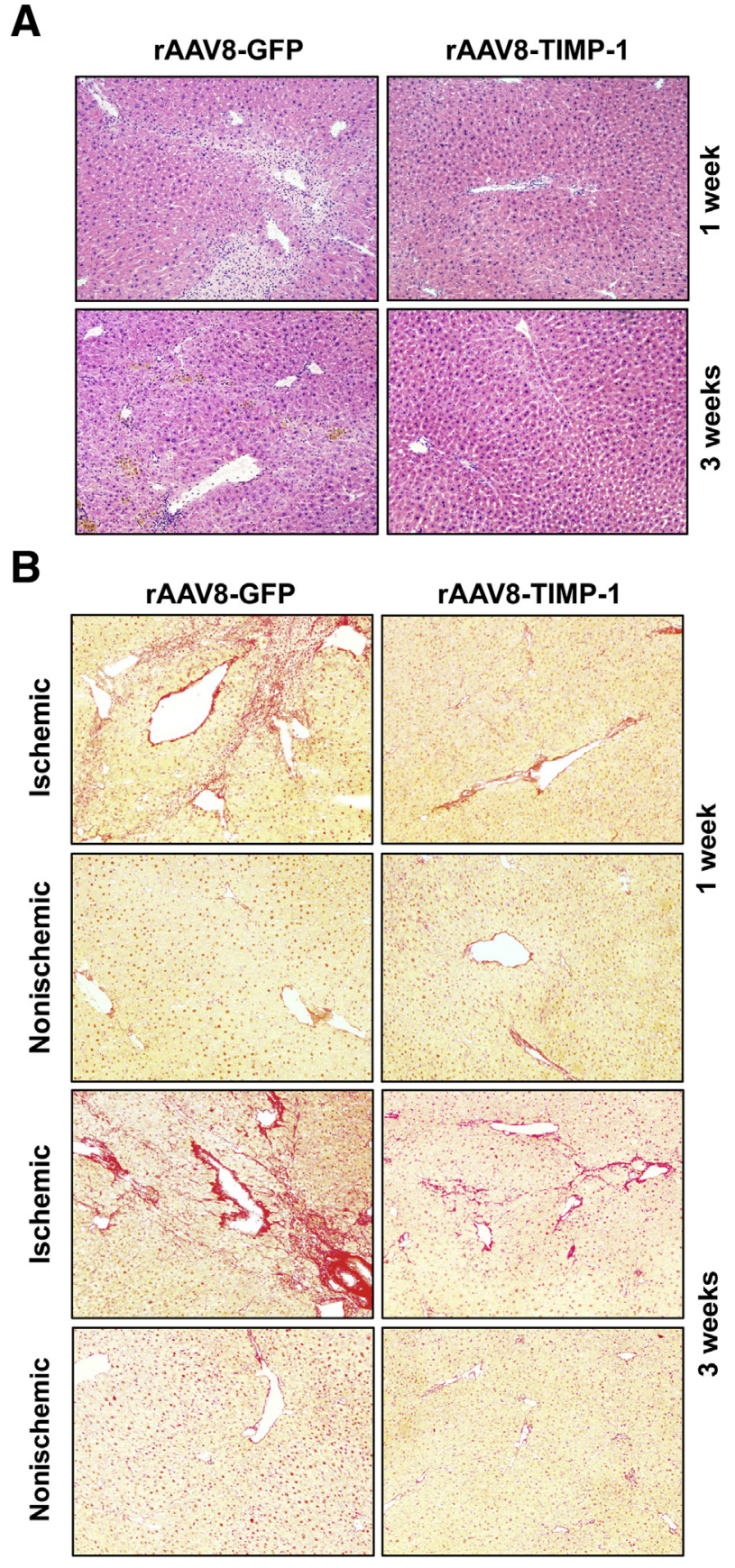

Figure 8 Long-term outcomes after hepatic ischemia and reperfusion injury (IRI). A: Hematoxylin and eosin staining of control and recombinant adenoassociated virus type 8 (rAAV8)-tissue inhibitor of metalloproteinases-1 (TIMP-1)-treated livers at 1 and 3 weeks after hepatic IRI; long-term rAAV8-TIMP-1-treated livers recover an almost normal architecture without visible signs of inflammatory infiltrate, particularly at 3 weeks after IRI. B: Sirius Red staining of control and rAAV8-TIMP-1-treated livers after 1 and 3 weeks of IRI; the ischemic lobes of rAAV8-TIMP-1-treated livers show significantly less collagen deposition than respective controls. The nonischemic lobes of control and rAAV8-TIMP-1-treated livers show virtually no collagen synthesis. $n=4$ per group (A and B). Original magnification, $\times 10$. GFP, green fluorescent protein.
(Figure 6, C and D). Plotting the percentage of Sytox ${ }^{+}$cells against their corresponding nuclear area shows that PMA and iH-GFP CM induced large neutrophil nuclear DNA expansions $\left(>300 \mu \mathrm{m}^{2}\right)$, which reflects NET release. In contrast, NET release was significantly reduced when neutrophils were treated with either $\mathrm{Cl}$-amidine or iH-TIMP-1 $\mathrm{CM}$ in vitro. In this case, most Sytox ${ }^{+}$cells were of smaller nuclear areas, consistent with neutrophils undergoing other forms of cell death (Figure 6, E and F). Murine rTIMP-1 is capable of reducing neutrophil-derived MMP-9 activity (Supplemental Figure S1). Next, rTIMP-1 was used to further assess the direct effects of TIMP-1 on NET release. Neutrophils were stimulated with PMA in the absence or presence of rTIMP-1, and NET release was evaluated by $\mathrm{Cit}-\mathrm{H} 3 / \mathrm{Ly}-6 \mathrm{G}^{+}$cell quantification. The percentage of Cit$\mathrm{H} 3 / \mathrm{Ly}^{-6 \mathrm{G}^{+}}$cells was significantly depressed in PMAstimulated neutrophils treated with rTIMP-1 (Figure 7A). Moreover, Sytox green monitoring of NET formation revealed large areas of Sytox ${ }^{+}$DNA released from PMAstimulated neutrophils, which were significantly depressed in the presence rTIMP-1 (Figure 7, B-E). All together, these data provide evidence that TIMP-1 has the ability to reduce NET formation in a direct manner and support that TIMP-1 overexpression in hepatocytes can limit the impact of NET-mediated cytotoxicity.

\section{AAV-Mediated TIMP-1 Overexpression Ameliorates Histologic Changes in Long-Term Livers after IRI}

Hepatic IRI is also a major determinant of late liver histologic abnormalities. ${ }^{34}$ In parallel studies, the effect of TIMP-1 overexpression was assessed in the histology of long-term livers after IRI. Livers treated with rAAV8-TIMP-1 had a clear histologic improvement in the ischemic lobes at both 1 and 3 weeks after IRI, compared with long-term control livers, which showed large necrotic areas surrounded by intense concentrations of inflammatory cells (week 1) and significant collagen deposition (week 3) in the ischemic lobes (Figure 8, A and B). Excess of collagen deposition is often associated with delayed/ impaired liver regeneration and fibrotic changes. The nonischemic liver lobes of both rAAV8-TIMP-1-treated and control mice had negligible collagen deposition (Figure 8B).

\section{rAAV8-TIMP1 Gene Transfer Abrogates Lethal Hepatic IRI in TIMP1-Deficient Mice}

TIMP-1 deficiency renders mice vulnerable to extensive liver damage and subsequent animal death after hepatic IRI. ${ }^{13}$ Therefore, it was tested whether rAAV8-TIMP-1 gene therapy could rescue $T I M P-1^{-1-}$ mice from lethal hepatic IRI. The rAAV8-TIMP-1 vectors restored TIMP-1 expression in livers of $T I M P-1^{-1-}$ mice (Figure 9, A and B) and improved their liver function after IRI (Figure 9C). The rAAV8-TIMP1 therapy ameliorated the extensive histologic damage otherwise experienced by $T I M P-1^{-l-}$ livers after IRI 

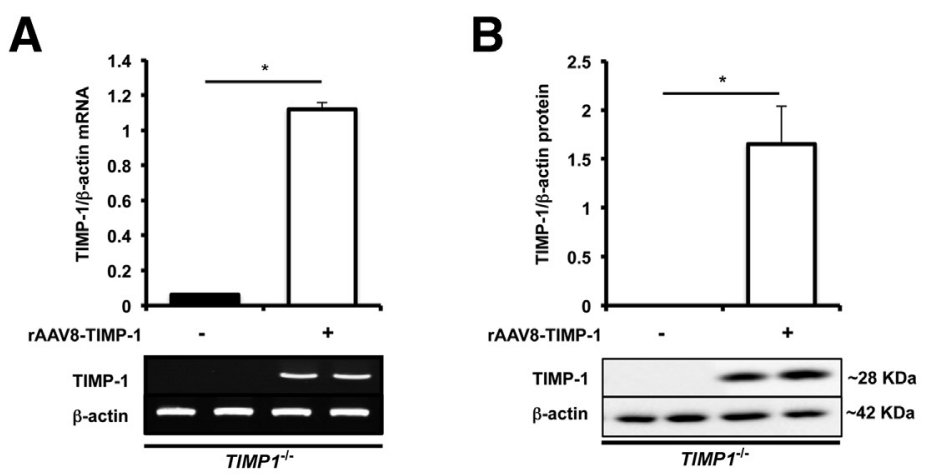

D

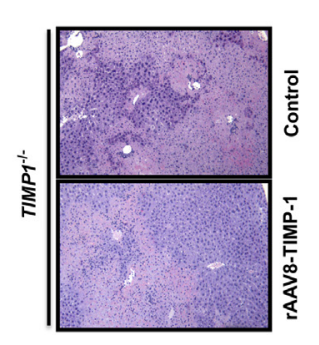

C
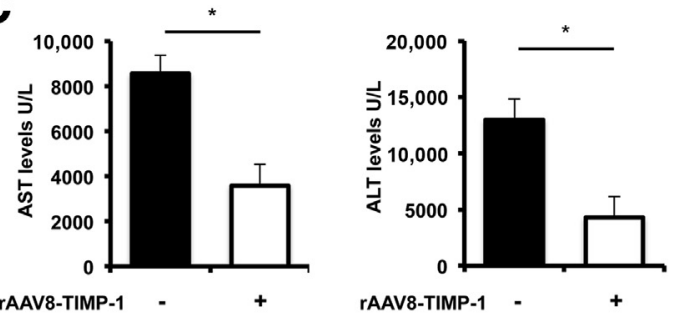

Figure 9 Adeno-associated virus type 8 (AAV8)-mediated transduction of tissue inhibitor of metalloproteinases-1 (TIMP1) in TIMP1 $^{-/}$mice. A-D: Recombinant AAV8 (rAAV8)TIMP-1 vectors restore TIMP-1 mRNA (A) and protein (B) expression in livers of TIMP1 ${ }^{-/-}$mice. TIMP1 ${ }^{-/-}$mice treated with rAAV8-TIMP-1 vectors have reduced transaminase levels (C) and improved liver histology at 6 hours after liver ischemia and reperfusion injury (D). E: The 7-day survival rate of TIMP1 ${ }^{-/-}$mice (red line-triangle) is enhanced by rAAV8-TIMP-1 vector therapy (blue line-diamond) from 50\% to $100 \%$. Open bars indicate rAAV8-TIMP-1-treated TIMP1-/livers; and closed bars, TIMP1-/- controls. $n=6$ per group $(\mathrm{A}-\mathrm{E}) .{ }^{*} P<0.05$. Original magnification, $\times 10$. ALT, alanine transaminase; AST, aspartate transaminase.
(Figure 9D). In addition, it increased the 7-day survival rate of TIMP $-1^{-1-}$ mice from $50 \%$ to $100 \%$ after surgery (Figure 9E). All wild-type mice survived surgery. Taken together, these results substantiate an important hepatoprotective role for TIMP-1 overexpression in liver IRI.

\section{Discussion}

To investigate whether increased availability of TIMP-1 has hepatoprotective effects, AAV vectors, including cloned TIMP- 1 cDNA and those packaged into the virus, were used in a well-established model of liver IRI. TIMP1-deficient mice exhibit significantly increased MMP-9 activity levels and more severe liver IRI than wild-type mice. ${ }^{13}$ The present study expands these results and demonstrates, for the first time, that rAAV delivery of TIMP-1 before injury conferred significant anti-inflammatory effects in wild-type mice and abrogated lethal hepatic IRI in TIMP-1 ${ }^{-1-}$ mice.

MMP-9 is induced by infiltrating leukocytes in damaged livers, and its deletion inhibits leukocyte transmigration and profoundly ameliorates hepatic IRI. ${ }^{7,26}$ Nevertheless, inhibition of MMP-9 in vivo with a broad-spectrum gelatinase inhibitor is ineffective in hepatic IRI. ${ }^{7}$ In contrast to MMP9, MMP-2 is constitutively expressed in liver, and its loss exacerbates MMP-9 activity and sinusoidal endothelial cell death after hepatic IRI. ${ }^{3}$ The currently available MMP pharmacologic inhibitors are not selective for a given MMP (including gelatinases), have a wide range of targets, and are limited by unforeseen adverse effects. ${ }^{35}$ Inhibition of MMPs by increasing the endogenous levels of their physiological inhibitors may be an alternative strategy to treat certain pathologies. ${ }^{36}$ Hepatic IRI is likely one of those pathologies that can be potentially ameliorated by increasing TIMP-1 levels. TIMP-1 is a particularly potent endogenous inhibitor of MMP-9 $9^{14,15,37}$; however, its low bioavailability is insufficient to target an elevated MMP-9 activity after hepatic IRI. ${ }^{7}$ Hence, to enhance the levels of TIMP-1 in liver, AAV8 vectors were used that, in addition to the capsid proteins conferring high affinity for hepatocytes, ${ }^{24,38}$ were engineered under the control of the thyroxine-binding globulin promoter; the latter confers strong liver-specific transgene expression. ${ }^{30}$ It was confirmed that AAV8mediated gene delivery of TIMPI and GFP was restricted to the liver and virtually absent from kidneys, heart, spleen, intestine, and lungs. Hepatic TIMP-1 overexpression was preferentially located in the perivascular areas, with slightly higher intensity in the pericentral zone. This transduction zonation is possibly caused by unequal hepatic distribution of AAV receptors or fenestrae size/density and is independent of transgene expression and promoter activity. ${ }^{39}$ Overall, rAAV8-TIMP-1 vector-treated livers were characterized by a significant reduction in inflammation and tissue damage. In addition to hepatic IRI, TIMP-1 has been reported to be protective in several pathologies, including ischemic brain injury ${ }^{40}$ and atherosclerotic plaque rupture. ${ }^{41}$ However, TIMP-1 has also been associated with both increased and reduced development of liver fibrosis. ${ }^{42,43}$ Although protective strategies against liver IRI will not require chronic applications of TIMP-1 (and therefore will have reduced risk of inducing fibrosis), rAAV8-TIMP-1 vector-treated long-term livers had significant histologic improvement with negligible collagen deposition after IRI. Growing evidence currently points to inflammatory cells as 
initiators of fibrogenic responses, ${ }^{44}$ and TIMP-1, by being able to depress the initial recruitment of inflammatory cells (and tissue damage), can potentially interfere with the initiation of fibrosis after liver IRI.

Neutrophils are the first cells recruited to inflamed tissues and are key mediators of liver injury. ${ }^{45}$ They produce extracellular DNA webs, called NETs, within minutes after activation. ${ }^{32}$ Although NET formation (or NETosis) is likely projected to function as a host defense mechanism against bacterial infections, it has also been largely implicated in damaging sterile inflammation. ${ }^{46-48}$ Huang et al ${ }^{48}$ elegantly established that NET formation initiates inflammatory responses and exacerbates organ damage in liver IRI. The exposed histones and granular proteins in the NET fibers as well as the intricate webs of extracellular DNA released by dying neutrophils undergoing NETosis can accentuate inflammatory responses and trap platelets and other cells, leading to vascular congestion. ${ }^{48,49}$ Our results confirmed the presence of NETs in the damaged livers and show that TIMP-1 overexpression hampered NET formation after hepatic IRI. The data also show that recombinant TIMP-1 and TIMP1 -rich conditioned media, derived from primary hepatocytes transduced with rAAV8-TIMP-1, were both capable of significantly reducing the extent of NET formation by cultured neutrophils. NET formation relies on post-translational modifications of nucleosome histones to facilitate chromatin unfolding and decondensation. ${ }^{50}$ The dominant proteolytic activity in NET formation has been attributed to neutrophil elastase, in response to microbial stimuli. ${ }^{51}$ However, recent studies have shown that elastase-deficient neutrophils form NETs during sterile inflammation, thus highlighting the existence of other relevant proteases in NETosis. ${ }^{52}$ Conceivably, MMP-9 could be involved in chromatin decondensation, providing a target for TIMP-1 in NET formation. MMP-9 is stored in large quantities in neutrophil granules before activation, and it colocalized with citrullinated histone $\mathrm{H} 3$ in neutrophils infiltrating the liver ischemic lobes after reperfusion. However, it must be kept in mind that TIMP-1 has functions beyond MMP inhibition and that MMP-independent actions for TIMP-1 in preventing NETosis cannot be ruled out. Some studies, for example, have reported that TIMP-1 can affect cell survival and differentiation independent of its MMP inhibitory role. ${ }^{53,54}$ The potential mechanisms by which TIMP-1 interferes with NET formation are certainly of interest and deserve future investigations.

To our knowledge, this is the first study showing that TIMP-1 overexpression is hepatoprotective in liver IRI. The advances presented herein may help in the design of novel therapies to treat hepatic IRI.

\section{Acknowledgments}

We thank Dr. Gerald Lipshutz and Chuhong Hu (University of California, Los Angeles) for their help with adenoassociated virus vector generation.

\section{Supplemental Data}

Supplemental material for this article can be found at https://doi.org/10.1016/j.ajpath.2018.05.002.

\section{References}

1. Eltzschig HK, Eckle T: Ischemia and reperfusion: from mechanism to translation. Nat Med 2011, 17:1391-1401

2. Duarte S, Baber J, Fujii T, Coito AJ: Matrix metalloproteinases in liver injury, repair and fibrosis. Matrix Biol 2015, 44-46:147-156

3. Kato H, Duarte S, Liu D, Busuttil RW, Coito AJ: Matrix metalloproteinase-2 (MMP-2) gene deletion enhances MMP-9 activity, impairs PARP-1 degradation, and exacerbates hepatic ischemia and reperfusion injury in mice. PLoS One 2015, 10:e0137642

4. Parks WC, Wilson CL, Lopez-Boado YS: Matrix metalloproteinases as modulators of inflammation and innate immunity. Nat Rev Immunol 2004, 4:617-629

5. Moore C, Shen XD, Gao F, Busuttil RW, Coito AJ: Fibronectinalpha4beta1 integrin interactions regulate metalloproteinase-9 expression in steatotic liver ischemia and reperfusion injury. Am J Pathol 2007, 170:567-577

6. Kuyvenhoven JP, Molenaar IQ, Verspaget HW, Veldman MG, Palareti G, Legnani C, Moolenburgh SE, Terpstra OT, Lamers CB, van Hoek B, Porte RJ: Plasma MMP-2 and MMP-9 and their inhibitors TIMP-1 and TIMP-2 during human orthotopic liver transplantation: the effect of aprotinin and the relation to ischemia/reperfusion injury. Thromb Haemost 2004, 91:506-513

7. Hamada T, Fondevila C, Busuttil RW, Coito AJ: Metalloproteinase-9 deficiency protects against hepatic ischemia/reperfusion injury. Hepatology 2008, 47:186-198

8. Lingwal N, Padmasekar M, Samikannu B, Bretzel RG, Preissner KT, Linn T: Inhibition of gelatinase B (matrix metalloprotease-9) activity reduces cellular inflammation and restores function of transplanted pancreatic islets. Diabetes 2012, 61:2045-2053

9. Turner RJ, Sharp FR: Implications of MMP9 for blood brain barrier disruption and hemorrhagic transformation following ischemic stroke. Front Cell Neurosci 2016, 10:56

10. Bramwell KK, Mock K, Ma Y, Weis JH, Teuscher C, Weis JJ: betaGlucuronidase, a regulator of lyme arthritis severity, modulates lysosomal trafficking and MMP-9 secretion in response to inflammatory stimuli. J Immunol 2015, 195:1647-1656

11. Visse R, Nagase H: Matrix metalloproteinases and tissue inhibitors of metalloproteinases: structure, function, and biochemistry. Circ Res 2003, 92:827-839

12. Sternlicht MD, Werb Z: How matrix metalloproteinases regulate cell behavior. Annu Rev Cell Dev Biol 2001, 17:463-516

13. Duarte S, Hamada T, Kuriyama N, Busuttil RW, Coito AJ: TIMP-1 deficiency leads to lethal partial hepatic ischemia and reperfusion injury. Hepatology 2012, 56:1074-1085

14. O'Connell JP, Willenbrock F, Docherty AJ, Eaton D, Murphy G: Analysis of the role of the $\mathrm{COOH}$-terminal domain in the activation, proteolytic activity, and tissue inhibitor of metalloproteinase interactions of gelatinase B. J Biol Chem 1994, 269:14967-14973

15. Bjorklund M, Koivunen E: Gelatinase-mediated migration and in vasion of cancer cells. Biochim Biophys Acta 2005, 1755:37-69

16. Ries C: Cytokine functions of TIMP-1. Cell Mol Life Sci 2014, 71 659-672

17. Brew K, Nagase $\mathrm{H}$ : The tissue inhibitors of metalloproteinases (TIMPs): an ancient family with structural and functional diversity. Biochim Biophys Acta 2010, 1803:55-71

18. Morgunova E, Tuuttila A, Bergmann U, Tryggvason K: Structural insight into the complex formation of latent matrix metalloproteinase 2 with tissue inhibitor of metalloproteinase 2. Proc Natl Acad Sci U S A 2002, 99:7414-7419 
19. Muzyczka N: Use of adeno-associated virus as a general transduction vector for mammalian cells. Curr Top Microbiol Immunol 1992, 158: 97-129

20. Nakai H, Fuess S, Storm TA, Muramatsu S, Nara Y, Kay MA: Unrestricted hepatocyte transduction with adeno-associated virus serotype 8 vectors in mice. J Virol 2005, 79:214-224

21. Wang B, Li J, Xiao X: Adeno-associated virus vector carrying human minidystrophin genes effectively ameliorates muscular dystrophy in mdx mouse model. Proc Natl Acad Sci U S A 2000, 97: 13714-13719

22. Naldini L: Gene therapy returns to centre stage. Nature 2015, 526: $351-360$

23. Committee for the Update of the Guide for the Care and Use of Laboratory Animals: National Research Council: Guide for the Care and Use of Laboratory Animals: Eighth Edition. Washington, DC, National Academies Press, 2011

24. Hu C, Busuttil RW, Lipshutz GS: RH10 provides superior transgene expression in mice when compared with natural AAV serotypes for neonatal gene therapy. J Gene Med 2010, 12:766-778

25. Hu C, Lipshutz GS: AAV-based neonatal gene therapy for hemophilia A: long-term correction and avoidance of immune responses in mice. Gene Ther 2012, 19:1166-1176

26. Kato H, Kuriyama N, Duarte S, Clavien PA, Busuttil RW, Coito AJ: MMP-9 deficiency shelters endothelial PECAM-1 expression and enhances regeneration of steatotic livers after ischemia and reperfusion injury. J Hepatol 2014, 60:1032-1039

27. Duarte S, Kato H, Kuriyama N, Suko K, Ishikawa TO, Busuttil RW, Herschman HR, Coito AJ: Hepatic ischemia and reperfusion injury in the absence of myeloid cell-derived COX-2 in mice. PLoS One 2014, 9:e96913

28. Mook OR, Van Overbeek C, Ackema EG, Van Maldegem F, Frederiks WM: In situ localization of gelatinolytic activity in the extracellular matrix of metastases of colon cancer in rat liver using quenched fluorogenic DQ-gelatin. J Histochem Cytochem 2003, 51: $821-829$

29. Gonzalez AS, Bardoel BW, Harbort CJ, Zychlinsky A: Induction and quantification of neutrophil extracellular traps. Methods Mol Biol 2014, 1124:307-318

30. Yan Z, Yan H, Ou H: Human thyroxine binding globulin (TBG) promoter directs efficient and sustaining transgene expression in liver-specific pattern. Gene 2012, 506:289-294

31. Nathan C: Neutrophils and immunity: challenges and opportunities. Nat Rev Immunol 2006, 6:173-182

32. Brinkmann V, Reichard U, Goosmann C, Fauler B, Uhlemann Y, Weiss DS, Weinrauch Y, Zychlinsky A: Neutrophil extracellular traps kill bacteria. Science 2004, 303:1532-1535

33. Branzk N, Lubojemska A, Hardison SE, Wang Q, Gutierrez MG, Brown GD, Papayannopoulos V: Neutrophils sense microbe size and selectively release neutrophil extracellular traps in response to large pathogens. Nat Immunol 2014, 15:1017-1025

34. Henderson JM: Liver transplantation and rejection: an overview. Hepatogastroenterology 1999, 46 Suppl 2:1482-1484

35. Bauvois B: New facets of matrix metalloproteinases MMP-2 and MMP-9 as cell surface transducers: outside-in signaling and relationship to tumor progression. Biochim Biophys Acta 2012, 1825: $29-36$

36. Vandenbroucke RE, Libert C: Is there new hope for therapeutic matrix metalloproteinase inhibition? Nat Rev Drug Discov 2014, 13: 904-927
37. Gardner J, Ghorpade A: Tissue inhibitor of metalloproteinase (TIMP)-1: the TIMPed balance of matrix metalloproteinases in the central nervous system. J Neurosci Res 2003, 74:801-806

38. Sands MS: AAV-mediated liver-directed gene therapy. Methods Mol Biol 2011, 807:141-157

39. Bell P, Wang L, Gao G, Haskins ME, Tarantal AF, McCarter RJ, Zhu Y, Yu H, Wilson JM: Inverse zonation of hepatocyte transduction with AAV vectors between mice and non-human primates. Mol Genet Metab 2011, 104:395-403

40. Tejima E, Guo S, Murata Y, Arai K, Lok J, van Leyen K, Rosell A, Wang X, Lo EH: Neuroprotective effects of overexpressing tissue inhibitor of metalloproteinase TIMP-1. J Neurotrauma 2009, 26 : 1935-1941

41. de Vries MR, Niessen HW, Lowik CW, Hamming JF, Jukema JW, Quax PH: Plaque rupture complications in murine atherosclerotic vein grafts can be prevented by TIMP-1 overexpression. PLoS One 2012, 7:e47134

42. Wang H, Lafdil F, Wang L, Yin S, Feng D, Gao B: Tissue inhibitor of metalloproteinase 1 (TIMP-1) deficiency exacerbates carbon tetrachloride-induced liver injury and fibrosis in mice: involvement of hepatocyte STAT3 in TIMP-1 production. Cell Biosci 2011, 1:14

43. Osawa Y, Hoshi M, Yasuda I, Saibara T, Moriwaki H, Kozawa O: Tumor necrosis factor-alpha promotes cholestasis-induced liver fibrosis in the mouse through tissue inhibitor of metalloproteinase-1 production in hepatic stellate cells. PLoS One 2013, 8:e65251

44. Iredale JP, Pellicoro A, Fallowfield JA: Liver fibrosis: understanding the dynamics of bidirectional wound repair to inform the design of markers and therapies. Dig Dis 2017, 35:310-313

45. Jaeschke H, Hasegawa T: Role of neutrophils in acute inflammatory liver injury. Liver Int 2006, 26:912-919

46. Kaplan MJ, Radic M: Neutrophil extracellular traps: double-edged swords of innate immunity. J Immunol 2012, 189:2689-2695

47. Liu FC, Chuang YH, Tsai YF, Yu HP: Role of neutrophil extracellular traps following injury. Shock 2014, 41:491-498

48. Huang H, Tohme S, Al-Khafaji AB, Tai S, Loughran P, Chen L, Wang S, Kim J, Billiar T, Wang Y, Tsung A: Damage-associated molecular pattern-activated neutrophil extracellular trap exacerbates sterile inflammatory liver injury. Hepatology 2015, 62:600-614

49. Fuchs TA, Brill A, Duerschmied D, Schatzberg D, Monestier M, Myers DD Jr, Wrobleski SK, Wakefield TW, Hartwig JH, Wagner DD: Extracellular DNA traps promote thrombosis. Proc Natl Acad Sci U S A 2010, 107:15880-15885

50. Branzk N, Papayannopoulos V: Molecular mechanisms regulating NETosis in infection and disease. Semin Immunopathol 2013, 35:513-530

51. Papayannopoulos V, Metzler KD, Hakkim A, Zychlinsky A: Neutrophil elastase and myeloperoxidase regulate the formation of neutrophil extracellular traps. J Cell Biol 2010, 191:677-691

52. Martinod K, Witsch T, Farley K, Gallant M, Remold-O'Donnell E, Wagner DD: Neutrophil elastase-deficient mice form neutrophil extracellular traps in an experimental model of deep vein thrombosis. J Thromb Haemost 2016, 14:551-558

53. Guedez L, Martinez A, Zhao S, Vivero A, Pittaluga S, StetlerStevenson M, Raffeld M, Stetler-Stevenson WG: Tissue inhibitor of metalloproteinase 1 (TIMP-1) promotes plasmablastic differentiation of a Burkitt lymphoma cell line: implications in the pathogenesis of plasmacytic/plasmablastic tumors. Blood 2005, 105:1660-1668

54. Jackson HW, Defamie V, Waterhouse P, Khokha R: TIMPs: versatile extracellular regulators in cancer. Nat Rev Cancer 2017, 17:38-53 